\title{
Over-the-counter self-medication leading to intracranial hypertension in a young lady
}

\author{
A. M. Ramana Reddy, L. K. Prashanth, G. G. Sharat Kumar, G. Chandana, Rakesh Jadav \\ Advanced Neurosciences Institute, BGS Global Hospital, Bangalore, Karnataka, India
}

\section{ABSTRACT}

Intracranial hypertension (idiopathic-IIH and secondary) is a potentially treatable condition. Although various factors such as female gender and obesity, certain drugs have been implicated as risk factors for IIH, there remains a lack of clarity in the exact causal-effect relationship. In India, self-medication by obtaining drugs over the counter due to lack of adequate drug regulation and ignorance of the public is a very common practice with a potential for severe adverse effects. We present a case of a young lady who has developed intracranial hypertension possibly due to self-medication with steroids and cyproheptadine, obtained over the counter.

Keywords: Cyproheptadine, dexamethasone, idiopathic intracranial hypertension (IIH), intracranial hypertension, steroid withdrawal

\section{Introduction}

Headache is the one of the most common complaint in the neurology outpatient practice. Headache presenting with red flags like persistent vomiting, diplopia, blurring of vision, etc should raise suspicion of serious secondary causes of headache like raised intracranial pressure (ICP) prompting appropriate investigations. Idiopathic intracranial hypertension (IIH) is one such cause of raised ICP. The identification of IIH and work up for secondary causes of IIH will hep in deciding the most appropriate treatment for the patient. We present one such case of IIH with a rather surprising cause.

\section{Case Report}

A 26-year-old lady presented with chief complaint of headache of 4 months duration. It was off and on initially and then became continuous with increase in severity. One week, prior to presentation, the headache

\begin{tabular}{|l|l|}
\hline \multicolumn{2}{|c|}{ Access this article online } \\
\hline Quick Response Code: & Website: \\
\hline & www.ruralneuropractice.com \\
\cline { 2 - 2 } & \\
\hline
\end{tabular}

worsened in severity associated with transient blurring of vision. She had no other focal neurological or systemic symptoms. Past history revealed consuming dexamethasone $(0.5 \mathrm{mg} /$ day $)$ and cyproheptadine (4 mg/day) tablets for intentional weight gain, obtained over the counter without any prescription. She used the tablets for a period of 1 year and stopped abruptly 6 months back. However, she has not developed any acute symptoms of steroid withdrawal. The headache had started around 2 months after she stopped the medications.

At the time of evaluation, she weighed $53 \mathrm{~kg}$. Her height was $143 \mathrm{~cm}$, body mass index (BMI) was 25.9 (18.5-25: Normal; 25-29.9: Overweight; >30: Obese). Her ideal body weight (Devine formula) was $45.9 \mathrm{~kg} .{ }^{[1]} \mathrm{Her}$ blood pressure was $110 / 80 \mathrm{mmHg}$. Rest of the systemic examination was unremarkable. Examination revealed visual acuity of $6 / 6$ in both eyes with normal intraocular pressure. Fundus showed bilateral optic disc edema with splinter hemorrhage in the left eye [Figure 1]. There were no cotton wool spots/macular edema. Visual field charting by Humphrey perimeter showed enlarged blind spot bilaterally with superior and inferior areas of depression in the right eye. Rest of the neurological examination was normal. In view of the gradually progressive headache and bilateral optic disc edema, raised intracranial pressure was considered and evaluated for the same.

\section{Address for correspondence:}

Dr. A. M. Ramana Reddy, Consultant Neurologist, BGS Global Hospital, 67, Uttarahalli Road, Kengeri, Bangalore - 560060 , Karnataka, India. E-mail: drramana_am@yahoo.com 
Complete blood counts, erythrocyte sedimentation rate, blood sugar, renal and liver function tests, serum electrolytes, HIV, Venereal disease research laboratory test (VDRL), thyroid function tests were within normal limits. Brain magnetic resonance imaging (MRI) showed tortuosity of the bilateral optic nerves with prominence of the subarachnoid space around the optic nerves, flattening of the posterior sclera, partial empty sella [Figure 2]. There was no intracranial mass lesion. The lateral ventricles were small and symmetrical. MR venography did not show any occlusion/thrombosis. After brain MRI, diagnosis of idiopathic intracranial hypertension was considered. Lumbar puncture revealed a high opening pressure of $280 \mathrm{~mm} \mathrm{H}_{2} \mathrm{O}$ and normal cerebrospinal fluid (CSF) (clear, colorless, white blood cell count 2 , red blood cell 0 , protein $14.5 \mathrm{mg} / \mathrm{dL}$, glucose $76 \mathrm{mg} / \mathrm{dL}$, chloride $114 \mathrm{meq} / \mathrm{dL}$, India ink-negative). Following drainage of $20 \mathrm{~mL}$ of CSF, there was a significant reduction in the severity of headache lasting 2-3 days. A diagnosis of intracranial hypertension was made based on modified Dandy criteria. ${ }^{[2]}$ She was
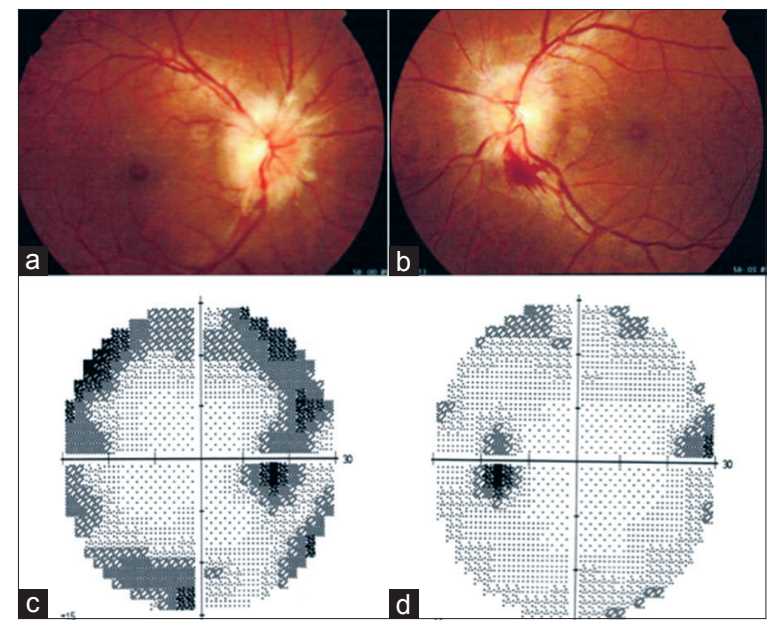

Figure 1: (a) Right fundus showing papilledema, (b) left fundus showing papilledema with splinter hemorrhage, (c) right eye showing enlargement of blind spot with superior and inferior areas of depression, (d) left eye enlargement of blind spot started on acetazolamide $250 \mathrm{mg}$ TID and advised $5 \%-10 \%$ weight loss. She has attained a weight loss of $8 \mathrm{~kg}(15 \%)$ over 6 months by dietary control and is completely asymptomatic now. Patient's prior approval was obtained for academic publication.

\section{Discussion}

Quinke first described the syndrome of increased intracranial pressure without ventriculomegaly or mass lesion in 1893. ${ }^{[3]}$ The earlier terms such as pseudotumorcerebri, benign intracranial hypertension have now been abandoned. The nomenclature, pathophysiology, clinical features, diagnostic criteria, and investigations have been extensively reviewed elsewhere and briefly discussed here. ${ }^{[2,4-9]}$ The current favored nomenclature for "idiopathic" variety is idiopathic intracranial hypertension (IIH) and cases with an identifiable cause are labeled as "Intracranial hypertension secondary to (cause)" ${ }^{[2]}$ Although the disease can occur in all ages and both sexes, young obese women of childbearing age is the typical patient for IIH. ${ }^{[2]}$ The role of obesity in Asian population in causing IIH has been debated recently. ${ }^{[10]}$ Young nonobese women $(\mathrm{BMI}<30)$ have also a risk of developing IIH if there is a $5 \%-15 \%$ recent weight gain. ${ }^{[11]}$ Patients with intracranial hypertension present with headache, visual symptoms, nausea, vomiting, tinnitus and evidence of papilledema, constricted fields, or sixth nerve palsy on examination. ${ }^{[4]}$ Progressive permanent vision loss is the dreaded complication of IIH. ${ }^{[2,4,12]}$ Although various medications such as vitamin A over dosage, tetracyclines, and sudden withdrawal of steroids have been described to trigger intracranial hypertension, a consistent relationship is only seen with tetracyclines. . $^{[4,8,9,13-15]}$ Cyproheptadine has not been reported to cause intracranial hypertension. Its contribution in our case is debated. The MRI features of IIH include "empty sella," slit-like ventricles, posterior globe flattening, intraocular

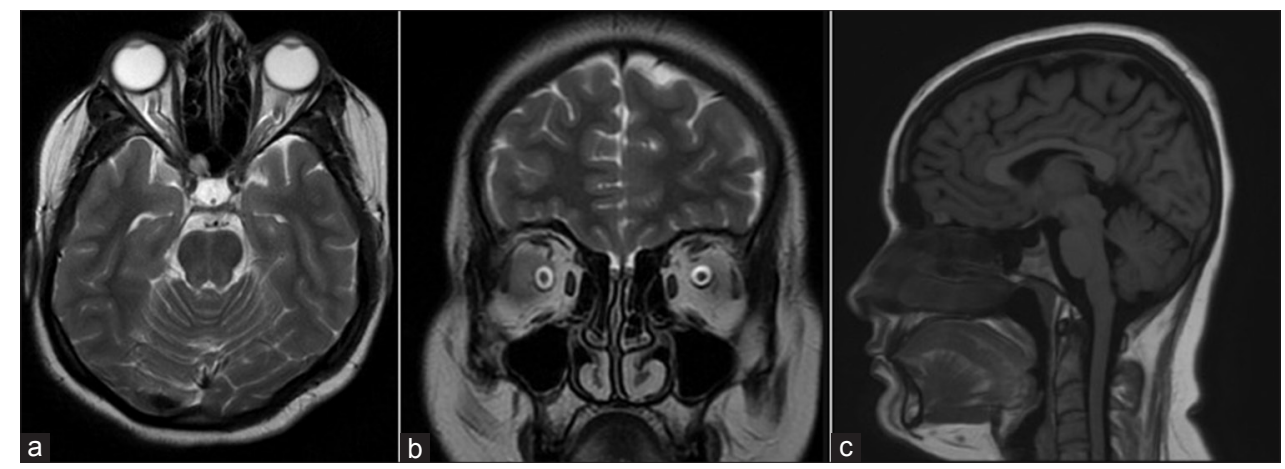

Figure 2: (a) T2-axial brain showing tortuosity of bilateral optic nerves along with prominent optic nerve sheath, (b) T2-coronal brain showing enlarged subarachnoid space around the optic nerve, (c) T1 sagittal showing empty sella 
protrusion, optic nerve sheath enlargement, optic nerve tortuosity, and transverse sinus narrowing ${ }^{[6]}$ The treatment of IIH begins with a lumbar puncture followed by weight loss and medical management, acetazolamide being the most commonly used drug. ${ }^{[2,5]}$ Weight loss of $5 \%-10 \%$ is a critical part of treatment of IIH. ${ }^{[5,16,17]}$ Other therapeutic options include venous sinus stenting, CSF diversion procedures, and surgery. ${ }^{[12,18]}$

In spite of the presence of all the above risk factors in our patient, their individual contribution to the clinical picture is not clear. Our patient had a borderline BMI of 25.9 (normal 18.5-24.9) and weighed $2.1 \mathrm{~kg}$ more than her ideal body weight. She had a weight gain of $10.4 \%$ over 1 year. Patients with this body profile are seen very often in general population, but rarely have IIH. What had caused IIH-like picture in our patient? Although the role of various mechanisms contributing to the development of IIH is debated, the most likely causative factor in this case is the recent weight gain in a young female of childbearing age. The individual contribution of cyproheptadine, prolonged steroid usage, and abrupt withdrawal remains rather unclear at this time.

This case raises two important concerns. There is still lack of clarity in the causal-effect relationship in IIH with reference to the role of obesity, weight gain, steroid usage, steroid withdrawal, and their individual contribution to the causation of IIH, which needs to be studied.

Another concern of utmost public health importance is the practice of self-medication and unregulated drug dispensing by the pharmacies. Self-medication is a very fairly common practice in India owing its roots to prevailing socioeconomic and cultural factors. However, the knowledge about the harmful effects of self-medication is very limited. The easy availability of potentially toxic drugs such as steroids over the counter is really alarming. Doctors and hospitals should educate the patients about harmful effects of self-medication. Effective use of mass media should be done to take the message to the masses. Drug regulatory authorities should strictly enforce laws pertaining to drug dispensing. Multipronged approach utilizing the media, government organizations, nongovernment organizations, doctors, pharmaceutical industry targeting the general population, as well as the pharmacy distributors should be used to increase the awareness about harmful effects of self-medication and control unregulated drug dispensing.

\section{References}

1. Pai MP, Paloucek FP. The origin of the "ideal" body weight equations. Ann Pharmacother 2000;34:1066-9.

2. Friedman DI, Jacobson DM. Idiopathic intracranial hypertension. J Neuroophthalmol 2004;24:138-45.

3. Grant DN. Benign intracranial hypertension. A review of 79 cases in infancy and childhood. Arch Dis Child 1971;46:651-5.

4. Ball AK, Clarke CE. Idiopathic intracranial hypertension. Lancet Neurol 2006;5:433-42.

5. Biousse V, Bruce BB, Newman NJ. Update on the pathophysiology and management of idiopathic intracranial hypertension. J Neurol Neurosurg Psychiatry 2012;83:488-94.

6. Degnan AJ, Levy LM. Pseudotumor cerebri: Brief review of clinical syndrome and imaging findings. AJNR Am J Neuroradiol 2011;32:1986-93.

7. Friedman DI, Jacobson DM. Diagnostic criteria for idiopathic intracranial hypertension. Neurology 2002;59:1492-5.

8. Friedman DI. Medication-induced intracranial hypertension in dermatology. Am J Clin Dermatol 2005;6:29-37.

9. Wall M. Idiopathic intracranial hypertension. Neurol Clin 2010;28:593-617.

10. Kim TW, Choung HK, Khwarg SI, Hwang JM, Yang HJ. Obesity may not be a risk factor for idiopathic intracranial hypertension in Asians. Eur J Neurol 2008;15:876-9.

11. Daniels AB, Liu GT, Volpe NJ, Galetta SL, Moster ML, Newman NJ, et al. Profiles of obesity, weight gain, and quality of life in idiopathic intracranial hypertension (pseudotumor cerebri). Am J Ophthalmol 2007;143:635-41.

12. Thambisetty M, Lavin PJ, Newman NJ, Biousse V. Fulminant idiopathic intracranial hypertension. Neurology 2007;68:229-32.

13. Ang ER, Zimmerman JC, Malkin E. Pseudotumor cerebri secondary to minocycline intake. J Am Board Fam Pract 2002;15:229-33.

14. Chiu AM, Chuenkongkaew WL, Cornblath WT, Trobe JD, Digre KB, Dotan SA, et al. Minocycline treatment and pseudotumor cerebri syndrome. Am J Ophthalmol 1998;126:116-21.

15. Kesler A, Goldhammer Y, Hadayer A, Pianka P. The outcome of pseudotumor cerebri induced by tetracycline therapy. Acta Neurol Scand 2004;110:408-11.

16. Ko MW. Idiopathic intracranial hypertension. Curr Treat Options Neurol 2011;13:101-8.

17. Wong R, Madill SA, Pandey P, Riordan-Eva P. Idiopathic intracranial hypertension: The association between weight loss and the requirement for systemic treatment. BMC Ophthalmol 2007;7:15.

18. Higgins JN, Cousins C, Owler BK, Sarkies N, Pickard JD. Idiopathic intracranial hypertension: 12 cases treated by venous sinus stenting. J Neurol Neurosurg Psychiatry 2003;74:1662-6.

How to cite this article: Ramana Reddy AM, Prashanth LK, Sharat Kumar GG, Chandana G, Jadav R. Over-the-counter self-medication leading to intracranial hypertension in a young lady. J Neurosci Rural Pract 2014;5:384-6.

Source of Support: Nil. Conflict of Interest: None declared. 